\title{
Investigated the hospital utilization and medical resource usage of haemophilia $A$ and $B$ in Taiwan: 2001-2010
}

\author{
Yu H. Yan ${ }^{1}$, Chih M. Kung ${ }^{2 *}$ \\ ${ }^{1}$ Department of Medical Research, Tainan Municipal Hospital, Tainan, Chinese Taipei \\ ${ }^{2}$ Department of Information Technology and Communication, Shih Chien University Kaohsiung Campus, Kaohsiung, Chinese Taipei; \\ *Corresponding Author: alex@cvig.org
}

Received 15 July 2013; revised 15 August 2013; accepted 1 September 2013

Copyright (C) 2013 Yu H. Yan, Chih M. Kung. This is an open access article distributed under the Creative Commons Attribution License, which permits unrestricted use, distribution, and reproduction in any medium, provided the original work is properly cited.

\section{ABSTRACT}

Aim: The purpose of this study was to explore the impact of attributes of medical care organizations and patients on medical resource utilization by hemophilia (hemophilia $A$ and hemophilia B; Hemophilia with musculoskeletal system and without). Methods: Hospitalization due to hemophilia (ICD9: 286.0 and 286.1) and hemophilia with musculoskeletal system (ICD9: 710.xx-739.xx) between 2001 and 2010 were identified from a national health insurance database. Calculated by patients and hospital characteristics, length of hospital stay, medical cost of hospitalization was analyzed. Results: Of the total of $\mathbf{2 1 5 0}$ patients, a systemic analysis of the results showed a mean patient age of $\mathbf{3 0 . 2}$ and average medical costs per patient of NT \$672,335 (US $\$ 21,010$ ). We found the mean cost of cases being significantly higher during the hemophilia A that in the hemophilia B (NT $\$ 708,620$ vs. NT $\$ 422,322, P<0.001$ ), and medical costs such as those for hemophilia with musculoskeletal system (NT $\$ 1,419,158)$ were significantly higher than comparison patients (NT \$463,889) $(P<$ $0.001)$. Conclusions: Taiwan operates a national insurance global budget payment system. Healthcare system budgetary pressures are exacerbated by increases in the costs of hemophilia treatment. Suggesting that Bureau of National Health Insurance (BNHI) should consider establishing official primary and secondary prophylaxis treatment and dose regimen (such as dose or tailored-dose) should consider establishing a more aggressive and comprehensive standard of care for hemophiliacs. It is believed that that will have a significant impact on future treatment for hemophiliacs in Taiwan.

Keywords: Hemophilia; Musculoskeletal System; National Health Insurance

\section{INTRODUCTION}

Hemophilia is a rare, devastating, inherited condition in which repeated bleeding leads to disability, pain and early death [1]. Hemophilia is an X-linked congenital bleeding disorder, with deficiency of coagulation factor VIII (hemophilia A, HA) or factor IX (hemophilia B, HB). Hemophilia patients often have hemarthrosis with severe joint pain, which results in joint immobility and subsequent muscle atrophy, joint contracture, and gait disturbance [2]. During the past 30 years, the life expectancy for individuals with haemophilia has significantly increased, largely because of the advances in medical care, the introduction and accessibility of factor replacement therapies, as well as improvements in the treatment of infectious diseases [3,4]. Although individuals with haemophilia have benefited from safer, more effective factor replacement products, these advances have been paralleled by substantial cost increases in the treatment for these patients [5].

In March 1995, Taiwan established a National Health Insurance (NHI) programmed, with approximately 23 million insured, covering over $99 \%$ of Taiwan's population. The National Health Insurance Law defined haemophilia as a catastrophic illness, exempting patients from a copayment requirement and assuring patient's access to adequate treatment. However, an optimal national treatment guideline has not yet been developed [6]. In Taiwan, the estimated mean prevalence (all severities) per 100,000 males was $6.7 \pm 0.1$ for HA and $1.2 \pm 0.1$ for 
$\mathrm{HB}$, respectively, and showed a growth of 0.1 per 100,000 males every 2 - 3 year, respectively. The proportion of annually NHI medical expense for congenital abnormality of coagulation is around $0.4 \%-0.5 \%$ [7]. As such, the care of hemophilic patients is costly $[8,9]$, urgently needed to allow for newer insights, based on reliable data sources into resource allocation, cost-utility analysis in the treatment of haemophilia. The objectives of this study were to: identify and compare the overall cost and medical resource utilization of both treatments in order to provide a baseline model for Taiwan's national insurance payment system and a reference for medical health-care policy in Taiwan and elsewhere.

\section{METHODS}

\subsection{Database}

The dataset was sourced from the National Health Insurance Research Database (NHIRD) for the period from 2001 to 2010. Initiated in 1995, Taiwan's NHI program is characterized by a single plan with the government as the sole insurer, comprehensive benefits, low co-payments, and free choice of healthcare providers from a widely-dispersed network. The NHI had 23.34 million members (an over $99.5 \%$ coverage rate) at the end of 2012. The NHIRD is published annually by the Taiwan National Health Research Institute, and contains the original claim data and registration files for all the enrollees under the NHI program.

\subsection{Study Sample}

The study was designed as a retrospective study. In total, 2150 patients hospitalized with a discharge diagnosis of HA and HB (International Classification of Disease, Ninth Revision, Clinical Modification (ICD-9-CM) codes 286.0 and 286.1) between January 2001 and December 2010 were selected from the NHIRD. Of these sampled patients, we identified 460 patients (27.2\%) who had other diseases of the musculoskeletal system, according to the ICD-9-CM Clinical Modification code 710-739 (Co morbid disorders were identified at the enrolled hospitalization). If a patient had $\geq 2$ hospitalizations within a 30-day period, they were regarded as the same episode and we only included the first hospitalization. The study cohort in terms of gender, age $(<18,18-$ $30,31-44,45-64$, and $>65$ years), urbanization level ( 5 levels; from 1 [most urbanized] to 5 [least urbanized]), a similar sex and age distribution. The mean age for these 2,150 patients was 30.2 ( \pm 23.0 years) years, with a range between 1 and 98 years.

\subsection{Statistical Analyses}

The SPSS statistical package (SPSS System for Windows, version 18.0). Pearson Chi-square tests were used to examine the gender, age, distributions of urbanization level, hospital level (Medical center, Regional hospital and Community hospital), and Division of both patients (HA and HB; Haemophilia with Musculoskeletal system and without musculoskeletal systems). We also performed a $t$-test survival analysis to medical expenditures and average length of stay (ALOS). A $P$-value of $<0.05$ was considered statistically significant.

\section{RESULTS}

Taiwan HA and HB were included in the study sample. Haemophilia numbers during the study period included 219 in 2001, 213 in 2003, 233 in 2005, 217 in 2007, and 233 in 2010 . The Length of hospital stays for 9 days, respectively. Hospitalization length decreased by $16 \%$ over the period studied. Table 1 illustrates the annual rise in total hospitalization costs for Haemophilia treatment during the 10-year period studied. Costs rose from NT \$ 80.25 million in 2001 to NT $\$ 225.89$ million in 2010 (Figure 1), with a total increase over the period of $181 \%$.

The distribution of patients and hospital characteris-

Table 1. 2001-2010 Medical Cost Statistics for Hemophilia patients under National Health Insurance programs.

\begin{tabular}{|c|c|c|c|c|c|}
\hline Year & Patients & Inpatient Days & Medical Cost (NT \$) & Length of hospital stay & Avg. Medical Cost (NT \$) \\
\hline 2001 & 219 & 2,030 & $80,253,820$ & 9.3 & 366,456 \\
\hline 2002 & 253 & 2,508 & $98,607,907$ & 9.9 & 389,755 \\
\hline 2003 & 213 & 2,014 & $99,639,851$ & 9.5 & 467,793 \\
\hline 2004 & 264 & 2,012 & $123,283,179$ & 7.6 & 466,982 \\
\hline 2005 & 233 & 1,957 & $165,386,702$ & 8.4 & 709,814 \\
\hline 2007 & 217 & 2,111 & $173,476,057$ & 9.7 & 799,429 \\
\hline 2008 & 276 & 2,625 & $245,403,021$ & 9.5 & 889,141 \\
\hline 2009 & 275 & 2,499 & $219,256,946$ & 9.1 & 797,298 \\
\hline 2010 & 223 & 1,744 & $225,895,896$ & 7.8 & $1,012,986$ \\
\hline Total average & 238 & 2,146 & $160,217,315$ & 9.0 & 672,335 \\
\hline
\end{tabular}




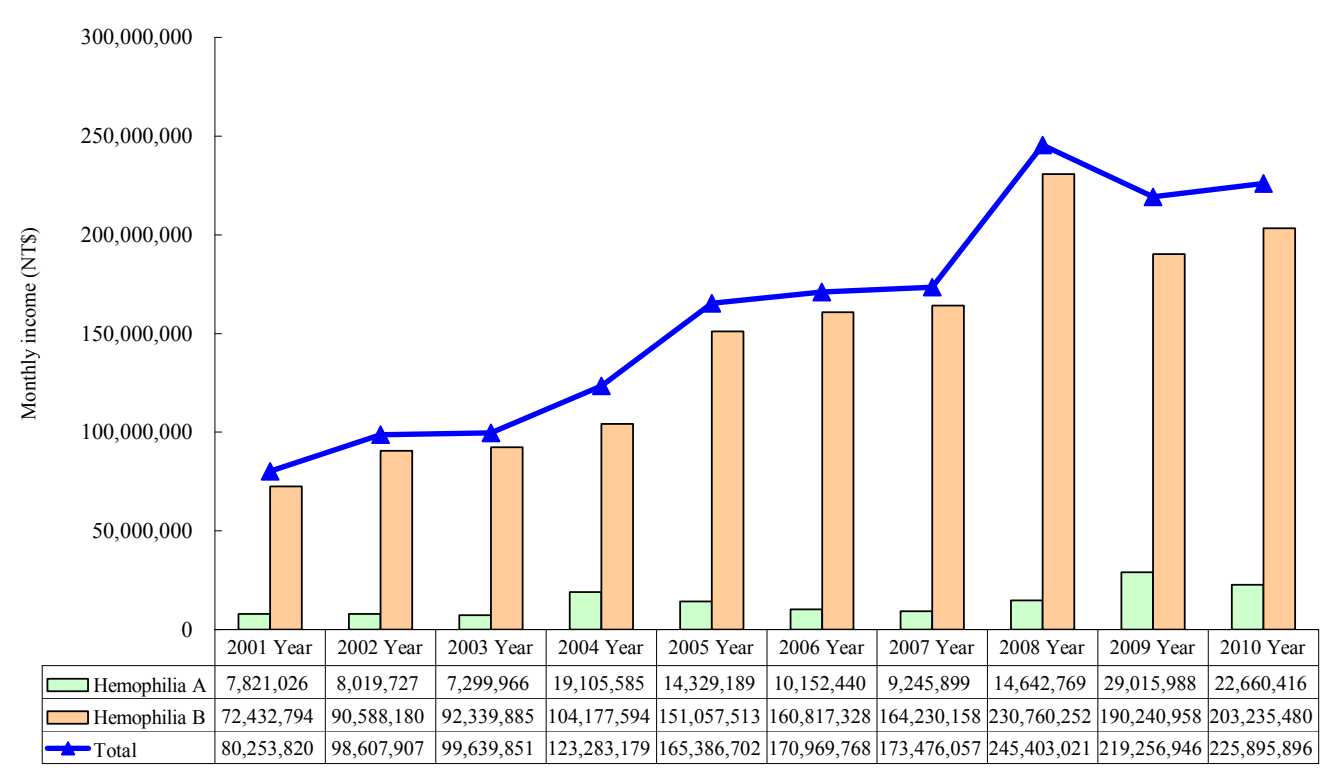

Figure 1. Total costs of Hemophilia patients.

tics between the study cohort and comparison cohort is presented in Table 2. Overall, 1965 (91.4\%) of the study participant were male and $185(8.6 \%)$ were female $(P<$ 0.05 ). This ratio supports the fact that the disease is more prominent amongst men. In terms of age, the average age of the participants was $30.2( \pm 23.0$ years $)$ years $(P<$ 0.001 ) years. Most were in the $<18$ age groups. This finding supports the fact that the disease is more prominent amongst the juvenile and children., with a range between 1 and 98 years, Clinical division in general medicine $(35.1 \%)$ and pediatrics $(35.1 \%)$ at most primary $(P<0.05)$. No significant difference in the lowincome families, hospital level and urbanization level was detected between the study cohort and comparison cohort.

Total costs are shown in Table 3. 85.9\% (patients 1847) to HA and only $14.1 \%$ (303 patients) to HB. In this study, we found the mean cost of cases being significantly higher during the HA that in the HB (NT \$708,620 \pm NT $\$ 1,414,988$ vs. NT $\$ 422,322 \pm$ NT $\$ 880,159$, respectively, $P<0.001)$. Researchers categorized medical cost variables according to the Bureau of National Health Insurance guidelines as follows: diagnosis costs (including costs of inpatient diagnosis and consultation), ward costs (including ward and nursing costs), tube feeding costs, laboratory costs (including complete blood cell, glutei oxaloacetic transaminase, glutamic pyruvic transaminase, cholestero1, blood urea nitrogen, sodium, potassium, chlorine, cardiac catheterization and coronary angiography costs), X-ray costs (e.g., chest posterior to anterior costs), therapeutic procedure costs (including costs for therapeutic treatment, temporary pacemakers and electrocardiogram monitoring), blood/plasma costs, hem dialysis costs (including costs of hem dialysis and peritoneal dialysis), special materials costs, drug costs, dispensing service costs (e.g., unit dose dispensing costs) and injection service costs. The two classic types of studies in medical research are $\mathrm{HA}$ and $\mathrm{HB}$, researchers found a significant statistical difference in diagnosis $(95 \% \mathrm{CI}=339-1141)$, word $(95 \% \mathrm{CI}=448-5848)$, laboratory $(95 \% \mathrm{CI}=658$ - 2010), $\mathrm{X}$-ray $(95 \% \mathrm{CI}=500$ $1522)$, blood/plasma $(95 \% \mathrm{CI}=112-425)$, Hem dialysis $(95 \% \mathrm{CI}=1007-2508), \operatorname{drug}(95 \% \mathrm{CI}=157,905-$ $391,158)$, Dispensing service $(95 \% \mathrm{CI}=106-300)$, psychotherapy $(95 \% \mathrm{CI}=17-90)$ and injection service costs $(95 \% \mathrm{CI}=126-333)(P<0.001)$. No significant difference in costs for tube feeding, therapeutic procedure, surgical, rehabilitation, anesthesia and special materials $(P>0.1)$. Medical costs such as those for HA were significantly higher than HB $(P<0.001)$.

Table 4 shows that there was no significant difference in two classic types of studies in medical research are Haemophilia with musculoskeletal system (21.3\%) and Comparison patients $(78.7 \%)$, researchers found a significant statistical difference in gender, age, diagnosis, tube feeding, laboratory, therapeutic procedure, surgical, rehabilitation, blood/plasma, anesthesia, special materials, drug and dispensing service costs $(P<0.001)$. No significant difference in costs for word, X-ray, hem dialysis, psychotherapy and injection service cost $(P>0.1)$, Medical costs such as those for Haemophilia with musculoskeletal system were significantly higher than comparison patients $(P<0.001)$.

\section{DISCUSSION}

No official Haemophilia patient registry exists yet in Taiwan. The only available data are gathered by the NHI 
Table 2. Demographic characteristics and Hemophilia patients in Taiwan 2001-2010 $(N=2,150)$.

\begin{tabular}{|c|c|c|c|c|c|c|c|}
\hline \multirow{2}{*}{ Characteristics } & \multicolumn{2}{|c|}{ Hemophilia A $(n=1847)$} & \multicolumn{2}{|c|}{ Hemophilia B $(n=303)$} & \multicolumn{2}{|c|}{ Total $(n=2150)$} & \multirow{2}{*}{$P$} \\
\hline & $n$ & $\%$ & $n$ & $\%$ & $n$ & $\%$ & \\
\hline \multicolumn{8}{|l|}{ Patients Characteristics } \\
\hline Gender & & & & & & & $<0.05$ \\
\hline Male & 1677 & $(90.8)$ & 288 & $(95.0)$ & 1965 & $(91.4)$ & \\
\hline Female & 170 & $(9.2)$ & 15 & $(15)$ & 185 & (8.6) & \\
\hline Age, Years & & & & & & & $<0.001$ \\
\hline$<18$ & 627 & $(33.9)$ & 161 & $(53.1)$ & 788 & $(36.7)$ & \\
\hline $18-30$ & 261 & $(14.1)$ & 46 & $(15.2)$ & 307 & $(14.3)$ & \\
\hline $31-44$ & 416 & $(22.5)$ & 41 & $(13.5)$ & 457 & $(21.3)$ & \\
\hline $45-64$ & 355 & $(19.2)$ & 35 & $(11.6)$ & 390 & $(18.1)$ & \\
\hline$>65$ & 188 & $(10.2)$ & 20 & (6.6) & 208 & $(9.7)$ & \\
\hline low-income families & & & & & & & 0.930 \\
\hline Yes & 38 & $(2.1)$ & 6 & $(2.0)$ & 44 & $(2.0)$ & \\
\hline No & 1809 & $(97.9)$ & 297 & $(98.0)$ & 2106 & $(98.0)$ & \\
\hline \multicolumn{8}{|l|}{$\begin{array}{c}\text { Hospital } \\
\text { Characteristics }\end{array}$} \\
\hline Hospital level & & & & & & & 0.404 \\
\hline Medical center & 1267 & $(68.6)$ & 203 & $(67.0)$ & 1470 & $(68.4)$ & \\
\hline Regional hospital & 527 & $(28.5)$ & 87 & $(28.7)$ & 614 & $(28.6)$ & \\
\hline Community hospital & 53 & (2.9) & 13 & $(4.0)$ & 66 & (3.1) & \\
\hline Urbanization level & & & & & & & 0.210 \\
\hline 1 (most urbanized) & 797 & $(43.2)$ & 133 & $(43.9)$ & 930 & $(43.3)$ & \\
\hline 2 & 893 & $(48.3)$ & 149 & $(49.2)$ & 1042 & $(48.5)$ & \\
\hline 3 & 29 & (1.6) & 5 & $(1.7)$ & 34 & $(1.6)$ & \\
\hline 4 & 115 & $(6.2)$ & 11 & (3.6) & 126 & $(5.9)$ & \\
\hline 5 (least urbanized) & 13 & $(0.7)$ & 5 & (1.7) & 18 & $(0.8)$ & \\
\hline Division & & & & & & & $<0.05$ \\
\hline General Medicine & 669 & $(36.2)$ & 86 & $(28.4)$ & 755 & $(35.1)$ & \\
\hline General Surgery & 449 & $(24.3)$ & 75 & $(24.8)$ & 524 & $(24.4)$ & \\
\hline Obstetrics \& Gynecology & 21 & (1.1) & 1 & $(0.3)$ & 22 & (1.0) & \\
\hline Pediatrics & 623 & $(33.7)$ & 132 & $(43.6)$ & 755 & $(35.1)$ & \\
\hline Other & 85 & (4.6) & 9 & (3.0) & 94 & (4.4) & \\
\hline
\end{tabular}

for claims purposes. The current study investigated the hospital utilization and medical resource usage of haemophilia patients in Taiwan, between the years 2001 to 2010. Our results showed that the costs of medical care for haemophilia hospitalization increased annually. When compared to 2001, the growth in 2010 rose by $181 \%$, which is similar to the results of Kern et al. In Taiwan, access to medical care for haemophilia is guaranteed under an independent budget (pooled for rare diseases) and separated from the capitalization budget for regular medical care. This approach assures haemophilia adequate treatment [6], and the rising medical care cost has exceeded all other diseases. In recent year (2012) for example, the population of major injuries and illness in Taiwan occupies only $3.86 \%$ of the National Health Insurance; however, they consume about $27.6 \%$ of the national medical resources, particularly with haemophilia patients being the highest resource users. Each haemophilia patient requires on average an annual expense of NT $\$ 3,115,426$, followed by respirator patients (NT $\$ 712,480 /$ year), di- 
Table 3. Hemophilia A and Hemophilia B on medical cost.

\begin{tabular}{|c|c|c|c|c|c|c|c|}
\hline \multirow{3}{*}{$\begin{array}{l}\text { Variables } \\
\text { Diagnosis }\end{array}$} & \multicolumn{2}{|c|}{ Hemophilia A $(n=1847)$} & \multicolumn{2}{|c|}{ Hemophilia B $(n=303)$} & \multirow{2}{*}{\multicolumn{2}{|c|}{$95 \% \mathrm{CI}$}} & \multirow{2}{*}{$P$} \\
\hline & Mean & SD & Mean & SD & & & \\
\hline & 3,667 & 4,007 & 2,927 & 3,157 & 339 & 1,141 & $<0.001$ \\
\hline Ward & 14,401 & 25,058 & 11,253 & 21,653 & 448 & 5,848 & $<0.05$ \\
\hline Tube feeding & 334 & 1,913 & 249 & 1,754 & 144 & 316 & 0.466 \\
\hline Laboratory & 5,232 & 7,798 & 3,898 & 5,088 & 658 & 2,010 & $<0.001$ \\
\hline X-ray & 2,318 & 5,499 & 1,306 & 3,938 & 500 & 1,522 & $<0.001$ \\
\hline Therapeutic procedure & 3,879 & 10,326 & 2,814 & 10,535 & 215 & 2,345 & 0.097 \\
\hline Surgical & 4,705 & 11,111 & 4,334 & 10,422 & 968 & 1,709 & 0.587 \\
\hline Rehabilitation & 279 & 1,963 & 265 & 1,781 & 221 & 249 & 0.899 \\
\hline Blood/plasma & 282 & 3,379 & 13 & 235 & 112 & 425 & $<0.001$ \\
\hline Hemodialysis & 3,214 & 13,232 & 1,457 & 3,957 & 1,007 & 2,508 & $<0.001$ \\
\hline Anesthesia & 2,379 & 5,010 & 2,175 & 4,537 & 397 & 805 & 0.475 \\
\hline Special materials & 5,751 & 17,939 & 4,473 & 17,073 & 888 & 3,443 & 0.231 \\
\hline Drug & 660,718 & $1,393,090$ & 386,186 & 866,098 & 157,905 & 391,158 & $<0.001$ \\
\hline Dispensing service & 871 & 943 & 667 & 770 & 106 & 300 & $<0.001$ \\
\hline Psychotherapy & 55 & 801 & 1 & 19 & 17 & 90 & $<0.05$ \\
\hline Injection service & 528 & 1,192 & 298 & 777 & 126 & 333 & $<0.001$ \\
\hline Medical cost & 708,620 & $1,414,988$ & 422,322 & 880,159 & 167,795 & 404,800 & $<0.001$ \\
\hline Length of hospital stay & 9.3 & 10.4 & 7.3 & 7.3 & 1.0 & 2.9 & $<0.001$ \\
\hline
\end{tabular}

alysis patients (NT \$585,816/year), and cancer patients (NT \$134,870/year). In comparison, the national medical costs per person, on average, are only NT \$24,399 [10].

Haemophilia is a sex-linked recessive hereditary disease and is mostly prevalent in males, with a prevalence rate of $1 / 10,000$ in newborns [11]. This disease is categorized into two factors: $\mathrm{A}$ and $\mathrm{B}$, which are due to deficiency in clotting factor VIII or IX. The majority of patients are haemophilia A, occupying about $80 \%-85 \%$ [12]. Similar to past literature, our results showed that males have the highest rate of haemophilia A (HA) at $85 \%$. HA patients have higher medical costs and lengths of hospital stay per case than HB patients, and have the highest increase in medicine cost per case (NT \$274,550), which differed from the results obtained by Gautier [13], which showed that there were no significant differences between HA and HB patients on lengths of hospital stay and medical cost. It is possible that such discrepancy is due to the study being targeted only to adults, while our study focused on patients of all age levels.

We believe that this comprehensive 10-year, population-based study represents the first of its kind to attempt to examine the association between the level of urbanization, hospital level and Haemophilia, However, our results did not show any significant differences, demonstrating that treatment of haemophilia in Taiwan has not yet been affected by rural-urban gaps and difference in medical care received.

Previous literature has also pointed out that haemophilia patients may develop minor joint conditions as early as during their infant phase [14,15], and the joint condition will worsen with age [16], further restricting the patient's daily activities and social participation [15, 17]. Our study grouped haemophilia patient's musculoskeletal system together and others to another group. Our results showed that about $20 \%$ of hospitalized patients are afflicted with musculoskeletal conditions as well, with significant differences in gender, age, lengths of stay and medical costs. On average, the medical cost per case was as high as NT $\$ 1,419,158$, with cost of medicine occupying the most at $95 \%$. This finding is similar to Ullman and Hoots [18]. Mostly because of the high cost of FVIII replacement. Individuals with this disease require lifetime therapy with clotting factor (factor VIII for patients with haemophilia A and factor IX for patients with haemophilia B), either on a routine basis with scheduled prophylaxis, or episodically to treat bleeding episodes [19].

Haemophilia is a very rare disease; the prevalence of severe haemophilia is only about 2 per 100,000 [20]. Even expensive therapies for rare diseases have a small public impact and therefore may be more highly valued 
Table 4. Hemophilia with Musculoskeletal system and without medical cost.

\begin{tabular}{|c|c|c|c|c|c|}
\hline \multirow{2}{*}{ Variables } & \multicolumn{2}{|c|}{ Hemophilia with Musculoskeletal system $(n=460)$} & \multicolumn{2}{|c|}{ Comparison patients $(n=1,690)$} & \multirow{2}{*}{$P^{\mathrm{a}}$} \\
\hline & Mean & $(\%) \mathrm{SD}$ & Mean & $(\%) \mathrm{SD}$ & \\
\hline \multicolumn{6}{|l|}{ Patients Characteristics } \\
\hline Gender & & & & & $<0.01$ \\
\hline Male & 436 & $(94.8)$ & 1,529 & $(90.5)$ & \\
\hline Female & 24 & $(5.2)$ & 161 & $(9.5)$ & \\
\hline Age, Years & & & & & $<0.001$ \\
\hline$<18$ & 120 & $(26.1)$ & 668 & $(39.5)$ & \\
\hline $18-30$ & 75 & $(16.3)$ & 232 & $(13.7)$ & \\
\hline $31-44$ & 144 & $(31.3)$ & 313 & $(18.5)$ & \\
\hline $45-64$ & 102 & $(22.2)$ & 288 & $(17.0)$ & \\
\hline$>65$ & 19 & $(4.1)$ & 189 & $(11.2)$ & \\
\hline Low-income families & & & & & .556 \\
\hline Yes & 11 & $(2.4)$ & 33 & $(2.0)$ & \\
\hline No & 449 & $(97.6)$ & 1,657 & $(98.0)$ & \\
\hline \multicolumn{6}{|c|}{ Medical Expenditures (NT \$) } \\
\hline Diagnosis & 4,159 & 3,723 & 3,401 & 3,941 & $<0.001$ \\
\hline Ward & 13,806 & 15,782 & 13,999 & 26,532 & .882 \\
\hline Tube feeding & 72 & 909 & 390 & 2,081 & $<0.001$ \\
\hline Laboratory & 5,619 & 6,737 & 4,888 & 7,676 & $<0.05$ \\
\hline X-ray & 1,848 & 4,051 & 2,264 & 5,611 & .136 \\
\hline Therapeutic procedure & 1,923 & 5,724 & 4,220 & 11,248 & $<0.001$ \\
\hline Surgical & 9,057 & 14,813 & 3,454 & 9,382 & $<0.001$ \\
\hline Rehabilitation & 577 & 2,249 & 195 & 1,837 & $<0.001$ \\
\hline Blood/plasma & 83 & 1,414 & 288 & 3,456 & $<0.05$ \\
\hline Hemodialysis & 3,577 & 14,695 & 2,801 & 11,654 & .233 \\
\hline Anesthesia & 3,471 & 5,320 & 2,046 & 4,795 & $<0.001$ \\
\hline Special materials & 15,405 & 32,299 & 2,894 & 9,333 & $<0.001$ \\
\hline Drug & $1,358,045$ & $1,985,638$ & 421,692 & $1,003,824$ & $<0.001$ \\
\hline Dispensing service & 1,036 & 956 & 789 & 907 & $<0.001$ \\
\hline Psychotherapy & 20 & 188 & 54 & 832 & .125 \\
\hline Injection service & 452 & 766 & 507 & 1,228 & .242 \\
\hline Medical cost & $1,419,158$ & $2,009,275$ & 463,889 & $1,023,208$ & $<0.001$ \\
\hline Length of hospital stay & 11.8 & 11.3 & 8.3 & 9.5 & $<0.001$ \\
\hline
\end{tabular}

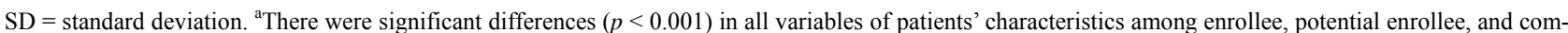
parison groups. The statistical difference was calculated by the $x^{2}$ test for categorical variables and by the t test for continuous variables.

[21]. Prophylaxis is recommended as optimal therapy for patients with severe haemophilia A by the World Federation of Haemophilia (WFH) and the World Health Organization (WHO) [22]. Preventive treatments such as FVIII prophylaxis, although expensive, may reduce the healthcare burden of chronic disease and may therefore be worth the cost [6]. In the long term, the definitions of prophylaxis reflect a wide spectrum of clinical conditions and objectives of the treatment, from the prevention of severe or life-threatening bleedings to the absence of naturopathy enabling patients to live a substantially normal life [6]. 
Our results provide more information about the value of haemophilia. In Taiwan, because of the available and advance health care system, life expectancy increased over the past decade. Although haemophilia is not a leading cause of death, it causes huge economic burden in society. Although the Bureau of National Health Insurance (BNHI) implemented a global budget project, and rare diseases have been allocated an independent budget for haemophilia, the author suggests that BNHI should consider establishing official primary and secondary prophylaxis treatment and dose regimen (such as dose or tailored-dose) should consider establishing a more aggressive and comprehensive standard of care for haemophilia. It is believed that that will have a significant impact on future treatment for haemophilia in Taiwan.

This study suffered from several limitations. The diagnoses of the conditions analyzed in this study were sourced from an administrative database using ICD-9$\mathrm{CM}$ codes. These diagnoses may be less accurate than those collected prospectively according to standardized procedures. Furthermore, in this study we only utilized discharge diagnoses. This strongly discounts the possibility that it would have been misdiagnosed as Haemophilia.

\section{REFERENCES}

[1] Feldman, B.M., Berger, K., Bohn, R., Carcao, M., Fischer, K., Gringeri, A., Hoots, K., Mantovani, L., Willan, A.R. and Schramm, W. (2012) Haemophilia prophylaxis: How can we justify the costs? Haemophilia, 18, 680-684. http://dx.doi.org/10.1111/j.1365-2516.2012.02790.x

[2] Chen, Y.C., Wei, T.S., Wu, T.J., Liu, C.C. and Shen, M.C. (2011) Correlation of joint symptoms and imaging studies among patients with hemophilia. Taiwan Journal of Physical Medicine and Rehabilitation, 39, 95-103.

[3] Dolan, G.., Hermans, C., Klamroth, R., Madhok, R., Schutgens, R.E.G. and Spengler, U. (2009) Challenges and controversies in haemophilia care in adulthood. Haemophilia, 15, 20-27.

http://dx.doi.org/10.1111/j.1365-2516.2008.01949.x

[4] Franchini, M. and Mannucci, P.M. (2010) Co-morbidities and quality of life in elderly persons with haemophilia. British Journal of Haematology, 148, 522-533. http://dx.doi.org/10.1111/j.1365-2141.2009.08005.x

[5] Khleif, A.A., Rodriguez, N., Brown, D. and Escobar. M.A. (2012) Utilization patterns and associated costs of factor assistance programmes among persons with haemophilia: A single institution review. Haemophilia, 18, e95-e100. http://dx.doi.org/10.1111/j.1365-2516.2011.02649.x

[6] Liou, W.S., Tu, T.C., Cheng, S.N., Chou, T.Y., Lee, C.F., Lin, T., Chung M.I. and Cham, T.M. (2011) Secondary prophylaxis treatment versus on-demand treatment for patients with severe haemophilia A: Comparisons of cost and outcomes in Taiwan. Haemophilia, 17, 45-54.

[7] Tu, T.C. (2012) Prevalence, incidence, medical resources consumption, related cost and outcomes comparisons of patients with haemophilia in Taiwan. Ph.D. Thesis, National University of Kaohsiung, Taiwan.

[8] Heemstra, H.E., Zwaan, T., Hemels, M., Feldman, B.M., Blanchette, V., Kern, M. and Einarson, T.R. (2005) Cost of severe hemophilia in Toronto. Hemophilia, 11, 254260. http://dx.doi.org/10.1111/j.1365-2516.2005.01082.x

[9] Kern, M., Blanchette, V., Stain, A.M., Einarson, T.R. and Feldman, B.M. (2004) Clnical and cost implications of target joints in Canadian boys with severe hemophilia A. The Journal of Pediatrics, 145, 628-634. http://dx.doi.org/10.1016/j.jpeds.2004.06.082

[10] Bureau of National Health Insurance (2013) Note: In 2012, for each NT\$100 of National Health Insurance payment, NT\$27 goes to the aid of patients of major illness and injuries.

[11] Srivastava, A., Brewer, A.K., Mauser-Bunschoten, E.P., Key, N.S., Kitchen, S., Llinas, A., Ludlam, C.A., Mahlangu, J.N, Mulder, K., Poon, M.C., Street, A. and Treatment Guidelines Working Group on Behalf of the World Federation of Hemophilia (2013) Guidelines for the management of hemophilia. Haemophilia, 19, e1-e47. http://dx.doi.org/10.1111/j.1365-2516.2012.02909.x

[12] Raffini, L. and Manno, C. (2007) Modern management of haemophilic arthropathy. British Journal of Haematology, 136, 777-787. http://dx.doi.org/10.1111/j.1365-2141.2007.06490.x

[13] Gautier, P., D’Alche-Gautier, M.J., Coatmelec, B., Marques-Verdier, A., Bertrand, M.A., Dieval, J. and Berthier, A.M. (2002) Cost related to replacement therapy during hospitalization in haemophiliacs with or without inhibitors: Experience of six French. Haemophilia, 8, 674-679. http://dx.doi.org/10.1046/j.1365-2516.2002.00671.x

[14] Rodriguez-Merchan, E.C. and Heim, M. (2005) Hemophilia orthopedic management with emphasis on developing countries. Seminars in Thrombosis and Hemostasis, 31, 518-526. http://dx.doi.org/10.1055/s-2005-922223

[15] Hacker, M.R., Funk, S.M. and Manco-Johnson, M.J. (2007) The Colorado haemophilia paediatric joint physical examination scale: Normal values and interrater reliability. Haemophilia, 13, 71-78. http://dx.doi.org/10.1111/j.1365-2516.2006.01387.x

[16] Dunn, A.L. (2005) Management and prevention of recurrent hemarthrosis in patients with hemophilia. Current Opinion in Hematology, 12, 390-394. http://dx.doi.org/10.1097/01.moh.0000169285.66841.c8

[17] Pipe, S.W. and Valentino, L.A. (2007) Optimizing outcomes for patients with severe haemophilia A. Haemophilia, 13, 1-16. http://dx.doi.org/10.1111/j.1365-2516.2007.01552.x

[18] Ullman, M. and Hoots, W.K. (2006) Assessing the costs for clinical care of patients with high responding factor VIII and IX inhibitors. Haemophilia, 12, 74-80. http://dx.doi.org/10.1111/j.1365-2516.2006.01370.x

[19] Escobar, M.A. (2010) Health economics in haemophilia: A review from the clinician's perspective. Haemophilia, 16, 29-34 http://dx.doi.org/10.1111/j.1365-2516.2010.02257.x

[20] Walker, I., Pai, M., Akabutu, J., Ritchie, B., Growe, G., Poon, M.C., Card, R., Ali, K., Israels, S., Teitel, J., et al. (1995) The Canadian hemophilia registry as the basis for 
a national system for monitoring the use of factor concentrates. Transfusion, 35, 548-551.

http://dx.doi.org/10.1046/j.1537-2995.1995.35795357875 $\underline{. x}$

[21] Hughes, D. A., Tunnage, B. and Yeo, S. T. (2005) Drugs for exceptionally rare diseases: Do they deserve special status for funding? Quarterly Journal of Medicine, 98, 829-836. http://dx.doi.org/10.1093/qjmed/hci128

[22] Berntorp, E., Boulyjenkov, V., Brettler, D., Chandy, M., Jones, P., Lee, C., Lusher, J., Mannucci, P., Peak, I. and Rickard, K. (1995) Modern treatment of haemophilia. Bulletin of the World Health Organization, 73, 691-701. 